Revista Sostenibilidad, Tecnología y Humanismo

36

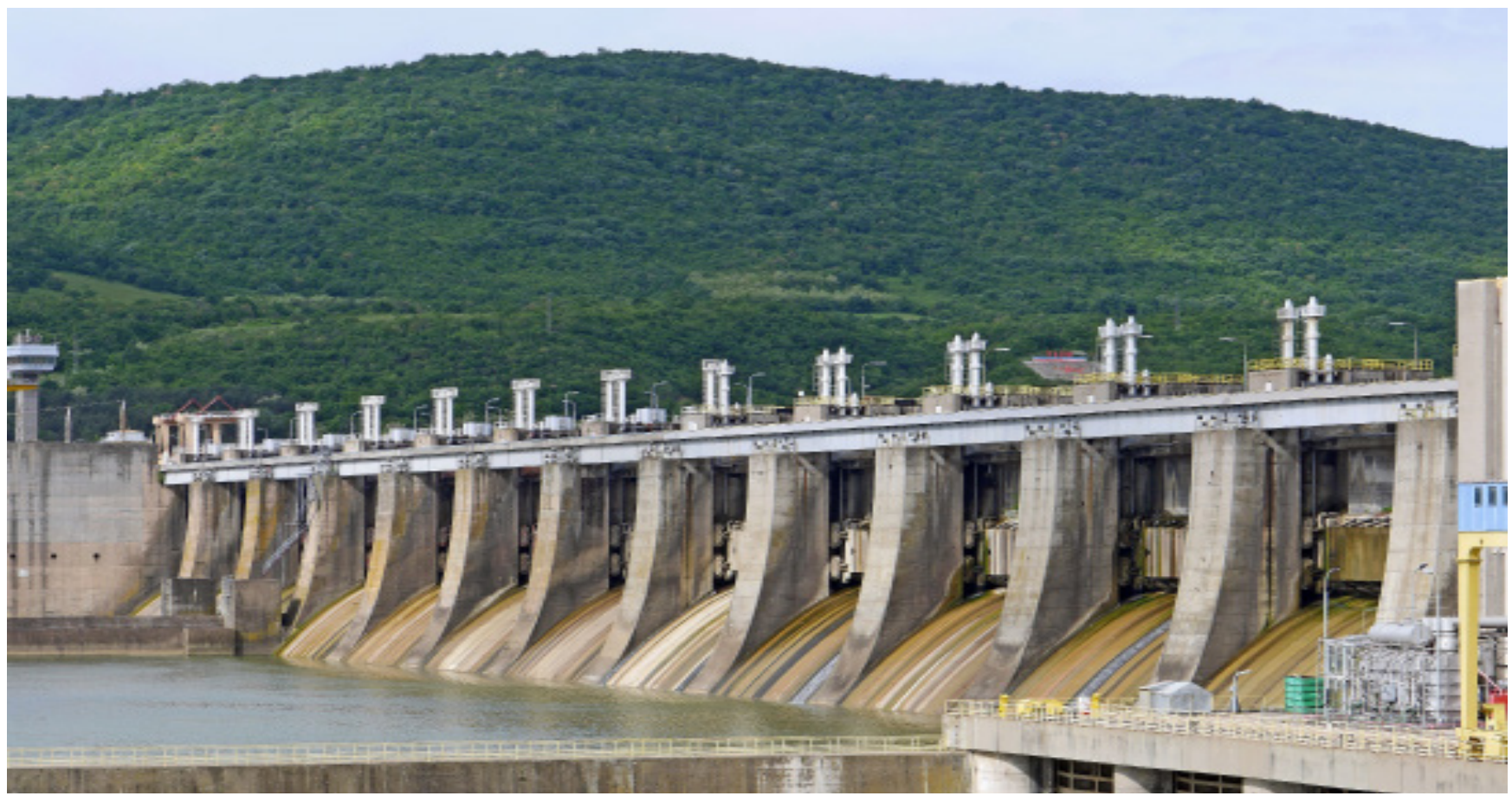

\title{
Análisis de estructuras para la retención de cantos rodados presentes en flujos torrenciales en zonas tropicales
}

\section{Analysis of structures for the retention of boulders present in torrential flows in tropical areas}

Yorly Cáceres-Paredes

Estudiante Ingeniería Civil, yorlycp@ufps.edu.co,

https://orcid.org/0000-0002-3104-3121, Universidad Francisco de Paula Santander, Cúcuta, Colombia
Nelson Javier Cely-Calixto

Magister en Obras Hidráulicas, nelsonjaviercc@ufps.edu.co,

https://orcid.org/0000-0002-2083-6978, Universidad Francisco de Paula Santander, Cúcuta, Colombia

Betsy Daniella Quintana-Ortíz

Estudiante Ingeniería Civil, betsydaniellaqo@ufps.edu.co,

https://orcid.org/0000-0003-0704-5677, Universidad Francisco de Paula Santander, Cúcuta, Colombia 
Resúmen

Los flujos de avalanchas son fenómenos naturales ocasionados por una erosión, un desprendimiento o la inestabilidad de un material depositado en el manto o corteza terrestre, causando grandes pérdidas debido a su poder destructivo siendo uno de los desastres naturales que más vidas ha cobrado, como en las áreas Andinas de Perú, Colombia, Ecuador, y Venezuela las cuales están entre las zonas más afectadas por esta clase de sucesos; es por esta razón, que existe la importancia de conocer los tipos de flujo que amenazan determinados sectores, y de esta manera poder implementar un sistema de disipación que se ajuste a sus características. El presente trabajo resume los tipos de flujos de avalanchas y los sistemas de mitigación y disipación de energía utilizados para contener el flujo y así alcanzar un menor impacto por parte del fenómeno, teniendo como base los resultados de búsqueda obtenidos a través de una recopilación bibliográfica extraídos de documentos investigativos como libros, proyectos de grado, artículos científicos, bases de suscripción y de libre acceso. Finalmente, s analiza cuáles son los dispositivos más eficientes e implementados a nivel internacional y nacional con respuestas favorables a los tipos de flujos más concurridos pertenecientes a las catástrofes que causan la remoción en masa de los diferentes sedimentos.

Palabras clave: Catástrofes, fenómeno, flujo de avalancha, mitigación, remoción en masa, sedimentos, sistema de disipación.

\section{Abstract}

Avalanche flows are natural phenomena caused by an erosion, a detachment or the instability of a material deposited in the mantle or earth's crust, causing great losses due to its destructive power being one of the natural disasters that has claimed more lives, as in the Andean areas of Peru, Colombia, Ecuador, and Venezuela which are among the most affected by this kind of events; it is for this reason that there is the importance of knowing the types of flows that threaten certain sectors, and thus be able to implement a dissipation system that fits their characteristics. The present work summarizes the types of avalanche flows and the mitigation and energy dissipation systems used to contain the flow and thus achieve a lower impact from the phenomenon, based on the search results obtained through a bibliographic compilation extracted from research documents such as books, degree projects, scientific articles, subscription and free access bases. Finally, s analyzes which are the most efficient and implemented devices at international and national level with favorable responses to the most concurred types of flows belonging to the catastrophes that cause the removal in mass of the different sediments

Keywords: Catastrophes, phenomenon, avalanche flow, mitigation, mass removal, sediments, dissipation system. 


\section{8}

\section{Introducción}

El principal interés del análisis de las propiedades de los tipos de flujos de avalanchas está orientado hacia el entendimiento de dichos sucesos en función de las partículas de arrastre y sedimentos presentes en su composición, para posteriormente elegir el sistema de disipación de energía más apto, de esta forma disminuir a gran escala el impacto que se puede generar a partir de estos fenómenos y contrarrestar la fuerza que estos alcanzan, por estos torrentes en zonas tropicales [1]. Los aluviones son producto del desprendimiento de material granular presente en las laderas, en conjunto con los efectos gravitatorios se produce el movimiento y transporte del mismo componente, haciendo que este cause un arrastre de los elementos que se encuentren a su paso [2], su recorrido está conformado por tres etapas denominadas zona de salida, de trayectoria y de llegada; donde desemboca el movimiento, donde se mantiene y donde finalmente desacelera para detener su desplazamiento [3]. De igual forma, estos eventos pueden estar compuestos por nieve, rocas, barro, ramas de árboles y tanto materia orgánica como inorgánica [4], dependiendo del lugar donde se origine estará integrado por diferentes elementos mezclados sin importar su composición ni el tamaño de sus partículas, teniendo en cuenta que cada tipo de material genera la clasificación del flujo de avalanchas [5].

En particular, debido a que la desembocadura de estos aludes está en la cima de las montañas, poseen unas pendientes muy altas generando así grandes velocidades y mayor capacidad de arrastre [6], de esta manera, la rapidez y densidad de la corriente está determinada por la cantidad de material transportado, este tipo de aluviones puede adquirir velocidades mayores a 20 metros por segundo, cuando el arrastre de lecho es mayor, el flujo es más denso y alcanza velocidades superiores [7], estas características tienen lugar en la etapa de propagación o trayectoria [8]. De esta manera, dependiendo de la afluencia y tipo de concentrados que posean, los sedimentos que se transportan causan grandes daños en diferentes ámbitos que varían entre lo material, lo socioeconómico, lo ambiental, y la vida humana, es decir, al obtener importantes proporciones de material se produce mayor deterioro en las diferentes poblaciones, generando situaciones catastróficas [9].

Por otra parte, al observar la tipología de las causas generadoras de lavas torrenciales en zonas tropicales, estas pueden proceder de diversas formas naturales, particularmente tienen como principal factor afectante y fuente de inicio la lluvia [10], siendo esta la que mayor impacto causa, las fuertes precipitaciones incentivan la remoción de los sedimentos debido al grado de saturación que alcanzan, provocando el desprendimiento del material, independientemente si el lugar de origen se encuentra en un porcentaje de pendiente alta o baja [11]. Otro agente importante que contribuye a este tipo de catástrofes son los sismos, al perjudicar la estabilidad del terreno, cuando se ocasiona el fuerte movimiento de las placas tectónicas, generando el desprendimiento de las partículas lo que conlleva a la gran concentración de material, así mismo las erupciones volcánicas y tormentas [12].

A partir de este fenómeno, se ha registrado a través de la historia un sin número de consecuencias y desastres que ha dejado a su paso, desde lesiones personales tanto físicas como psicológicas hasta pérdidas masivas de vidas humanas, afectando a su vez la existencia de la fauna y flora, produciendo un fuerte impacto ambiental, no obstante, el daño que originan en la infraestructura y orden territorial representa un gran perjuicio tanto económico como social generando un desbalance en la comunidad [13]. Debido a estas pérdidas continuas, el hombre ha trabajado por años en 
dispositivos de alerta temprana y en sistemas de contención de aludes, este último se basa en disipar la energía del flujo de avalancha, mediante procesos y recursos que permiten contrarrestar o disminuir en gran escala los daños que estas corrientes causan a las diferentes poblaciones [14].

En términos generales, al hacer un enfoque a escala global se tiene que la región con mayor índice de tragedias debido a este fenómeno es la región andina con cuatro principales países que tienen el mayor rating, los cuales son Ecuador, Venezuela, Perú, y Colombia, siendo estos dos últimos los que encabezan la lista con mayor grado de afectación [15]. Es importante destacar, el aumento significativo que ha tenido la población colombiana y de la misma forma la urbanización en las diferentes ciudades de esta nación, sin embargo, existe una tendencia en habitar zonas de alto riesgo expuestas a desastres naturales, además, es evidente el aumento en la deforestación para lograr los anteriores objetivos, esto ha desencadenado durante varias décadas distintos acontecimientos como avalanchas e inundaciones que han afectado en gran magnitud los bienes económicos y personales de la población [16]. Así, por ejemplo, la tragedia de Armero en el año 1985 cobrando más de 20.000 vidas; el alud en San Carlo - Antioquia en el año 1990 dejando 1.200 damnificados, 12 personas desaparecidas, cuatro muertos y cientos de personas sin residencia [17]; el desastre originado por un terremoto en la cuenca del rio Páez en el año 1994, el cual generó una avalancha y 3000 deslizamientos de tierra arrebatando la vida a 1100 personas y 500 desaparecidas [18], sin embargo, en el año; para el año 2005 se presentó en Girón - Santander una catástrofe producto del desbordamiento del Rio de Oro dejando cerca de 996 viviendas destruidas, más de 50 muertos y elevadas pérdidas económicas [19]; y por último, la tragedia de Salgar - Antioquia ocurrida en el año 2015 terminando con la vida de al menos 60 personas y ocasionado un sinfín de damnificados [20].

El fundamento base de una barrera contra avalanchas es disminuir el impacto que ocasiona este fenómeno para evitar el alcance destructivo que puede generar pérdida en diferentes ámbitos de la sociedad. Aunque, "el objetivo principal de estos sistemas de protección no se centra únicamente en retener el material para que no se produzcan mayores daños pendientes abajo, sino que también busca dejar pasar el agua para que siga el flujo natural. Con la retención del material se evitarán problemas como el arrastre de suelo a su paso y también el estancamiento de futuros elementos de drenaje, tuberías, etc., por el que transcurra el agua que arrastraría el material" [21]. Por este motivo, a través del tiempo se han realizado diferentes estudios de laboratorio en distintos países con el objetivo de diseñar un sistema capacitado para este propósito, a partir de dichas pruebas se analizaron métodos rígidos y flexibles; las estructuras rígidas se componen por diques de concreto y acero, soportando esfuerzos ejercidos por las grandes cargas de sedimentos que estas pueden acumular, para luego transmitirlos a su cimentación, sin embargo no permiten que sus elementos sufran deformaciones importantes sin antes fallar, como por ejemplo los diques de hormigón o mampostería hidráulica, diques de gaviones, diques reticulares y de rastrillo, presa abierta de doble pilar y gran viga de arriostramiento a estribos, entre otros [22].

Por otra parte, tenemos las estructuras flexibles que de igual forma soportan la fuerza de la colisión y su diseño permite la deformación elástica de la malla resistiendo el peso que recibe, en consecuencia, hace que se vuelva más eficaz y eficiente. Actualmente es la más implementada, en base a esto, tenemos que, estas dos clasificaciones son un contraste entre lo ambiguo y lo actual, ya que a medida que 


\section{0}

ha transcurrido el tiempo se han dejado de implementar las obras rígidas, y se ha puesto en marcha el funcionamiento de las flexibles, siendo estas últimas las que han traído igual o mayores beneficios en distintas áreas, en cuanto a la construcción por su menor tiempo de ejecución, como también en el ámbito económico haciendo su accionamiento más accesible. De este modo, se tiene un mayor énfasis en la composición y funcionamiento de los distintos elementos que contiene este sistema, los cuales cada uno aporta una labor diferente para así lograr una óptima respuesta ante el fenómeno.

Cada uno de los distintos componentes cumple una cometida diferente; su estructura interceptora (con su esquema de red de aros y alambres de alta resistencia) es la que recibe la colisión de una forma directa generada por la masa de escombros permitiendo la deformación elástica y propagando dichas presiones a el fundamento 0 base de construcción. Su estructura de soporte la cual interactúa con la anterior al ser inmediatamente conectada, sosteniéndola con su base de distintos materiales, principalmente un conjunto de postales diseñados con variadas medidas según lo requerido. Sus elementos conectivos conformados por una serie de cuerdas, actuando como emisoras de cargas a la base de la fundación además de poseer los elementos que disipan la energía generada por la erosión, los cuales están distribuidos en distintos sentidos cumpliendo una función en específico dependiendo de su posición. Su dispositivo disipador de energía hace parte del cableado anteriormente mencionado, el cual se comporta de una manera elástica recibiendo la colisión impulsada por el flujo, controlando el paso del material y disminuyendo el impacto que puede causar tanto a la sociedad como a la intemperie. Su estructura de base o cimentación, la cual actúa como anclaje al suelo, haciendo el soporte entre el suelo y el sistema que recibe las fuerzas por la avalancha, compuestos por materiales con alto nivel de resistencia para dar estabilidad y confiabilidad a la obra. Su mecanismo de defensa contra la corrosión, desempeña su tarea como barrera protectora contra la abrasión de los diversos materiales que componen el sistema, brindando un mayor rendimiento, vida útil y resistencia de las piezas implementadas. Y por último su placa base, consiste en un componente especial conformado por un sensor, actuando en posición perpendicular para obtener la medida de los esfuerzos generados a cortante que son causados en el sistema por el flujo.

\section{Materiales y métodos}

Para el presente estudio, se realizó una investigación descriptiva y documental basada en los resultados de búsqueda obtenidos a través de una recopilación bibliográfica a nivel nacional e internacional [23], extraídos de documentos investigativos como libros, proyectos de grado, artículos científicos y periodísticos, bases de suscripción y de libre acceso [24]; con el fin de determinar cuales son las estructuras existentes que contribuyen a la disipación de los distintos flujos torrenciales, a su vez, definir el sistema que presente mayor rendimiento frente a los flujos generados en zonas tropicales.

\section{Resultados}

Tipos de flujos de avalanchas

Según la determinada cantidad y concentración de material que presente un flujo, se puede determinar el tipo de aluvión, cuando se encuentra una corriente de altas concentraciones de arcillas y limos hablamos de "flujos de lodos", debido a que se genera un material homogéneo por la proporción de partículas finas [25]; al estar conformado por partículas gruesas como enrocados, gravas y arenas mezclados con agua, hace referencia a "flujos de rocas" o también denominados "hiperconcentrados" 
[26]; así mismo, cuando la avenida torrencial es dominada por sedimentos de variada granulometría o escombros toma el nombre de "flujos de detritos"; estas denominaciones son referidas a avalanchas en zonas tropicales [27], sin embargo, existen otros tipos de aludes que se presentan en zonas frías, las cuales están determinadas por un fluente principalmente compuesto de nieve, "Se originan con la pérdida de estabilidad del manto de nieve causada ya sea por cambios mecánicos y físicos en la estructura interna e interacción entre los granos de la nieve como por solicitaciones externas ejercidas por el paso de algún esquiador, caída de cornisas, ondas explosivas, sismos, entre otras" [2].

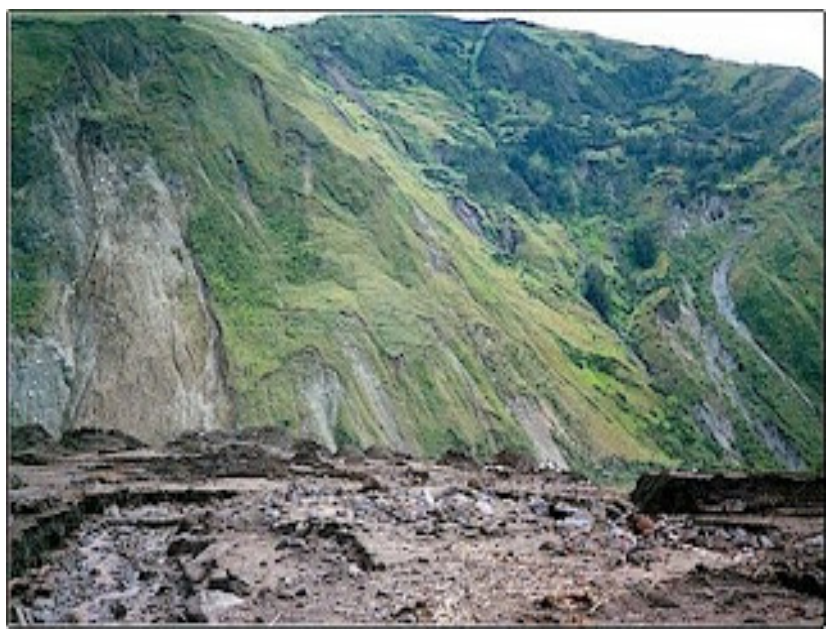

Figura. 1 Flujo de lodos [28]

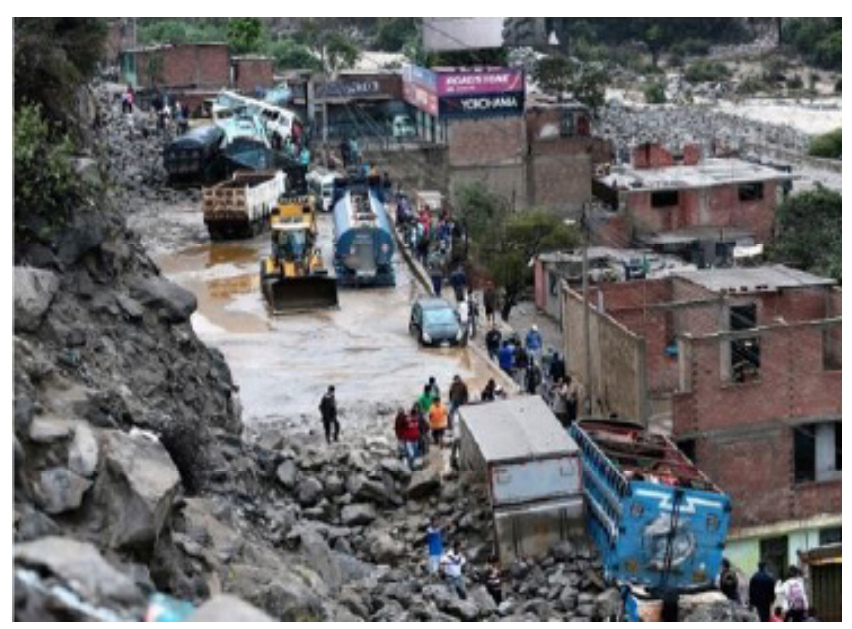

Figura. 2 Flujo hiperconcentrados [29]

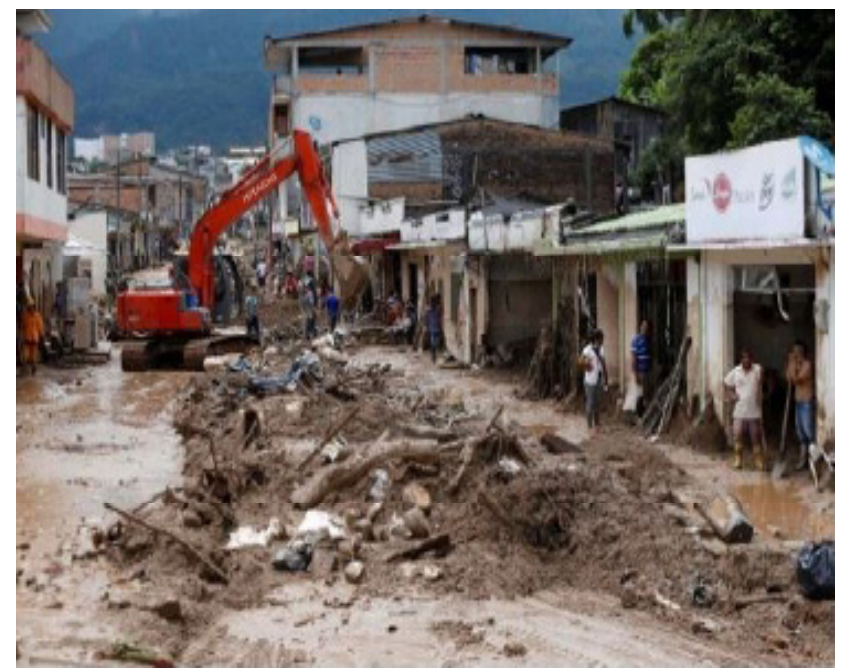

Figura. 3 Flujo de detritos [30]

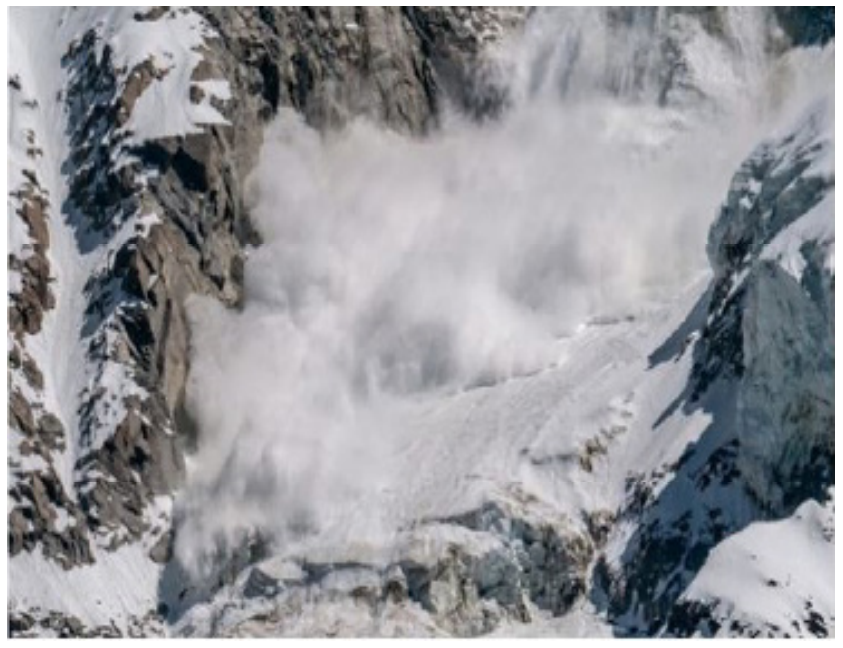

Figura. 4 Flujo de nieve [31]

Sistemas disipadores de energía en flujos de avalanchas para zonas tropicales

Respecto a sistemas de disipación de este fenómeno, existen investigaciones e implementaciones hechas en distintos países a partir de las fuertes pérdidas ocasionadas, teniendo como prioridad salvaguardar la vida humana y proteger el patrimonio de la sociedad, de igual modo [14] señalan que "el control de avalanchas no es otra cosa que una técnica que permite minimizar los daños que ellas causan al hombre, mediante una serie de acciones, actividades y elementos que modifican y/o minimizan los efectos finales producidos por ellas". Como resultado, 
durante años se han realizado cierta variedad de dispositivos con diferentes medidas y parámetros de diseño, en relación con las especificaciones presentes en cada lecho fluvial propenso a manifestar estos flujos de avalancha, observando sus principales falencias y comportamientos para que el resultado sea eficiente y se acondicione de manera exacta de acuerdo con la necesidad, se han implementado estructuras flexibles, rígidas y mixtos de prevención y disipación de fuerzas de acuerdo con sus características [32].

\section{Estructuras rígidas}

Las estructuras hidráulicas rígidas normalmente se construyen en concreto armado, concreto ciclópeo, gaviones y perfiles de hierro o acero, estas pueden clasificarse en dos tipos, cerradas o impermeables y abiertas o permeables; las primeras obstruyen el paso de la mayor proporción de sedimentos transportados, excepto las partículas de menor tamaño que pasan cuando el flujo desborda la estructura; las segundas poseen unas ranuras en el cuerpo del dique permitiendo el paso de solidos finos e interceptando solo los de mayor tamaño, ya que estos son los que ocasionan grandes impactos en las edificaciones aguas abajo, de esta forma se favorece el trayecto normal del flujo y de los distintos peces presentes en el lugar, además la capacidad de almacenamiento de este tipo de sistemas puede durar más tiempo [33].

Tabla 1. Sistemas ríqidos disipadores de eneraía en fluios de avalanchas.

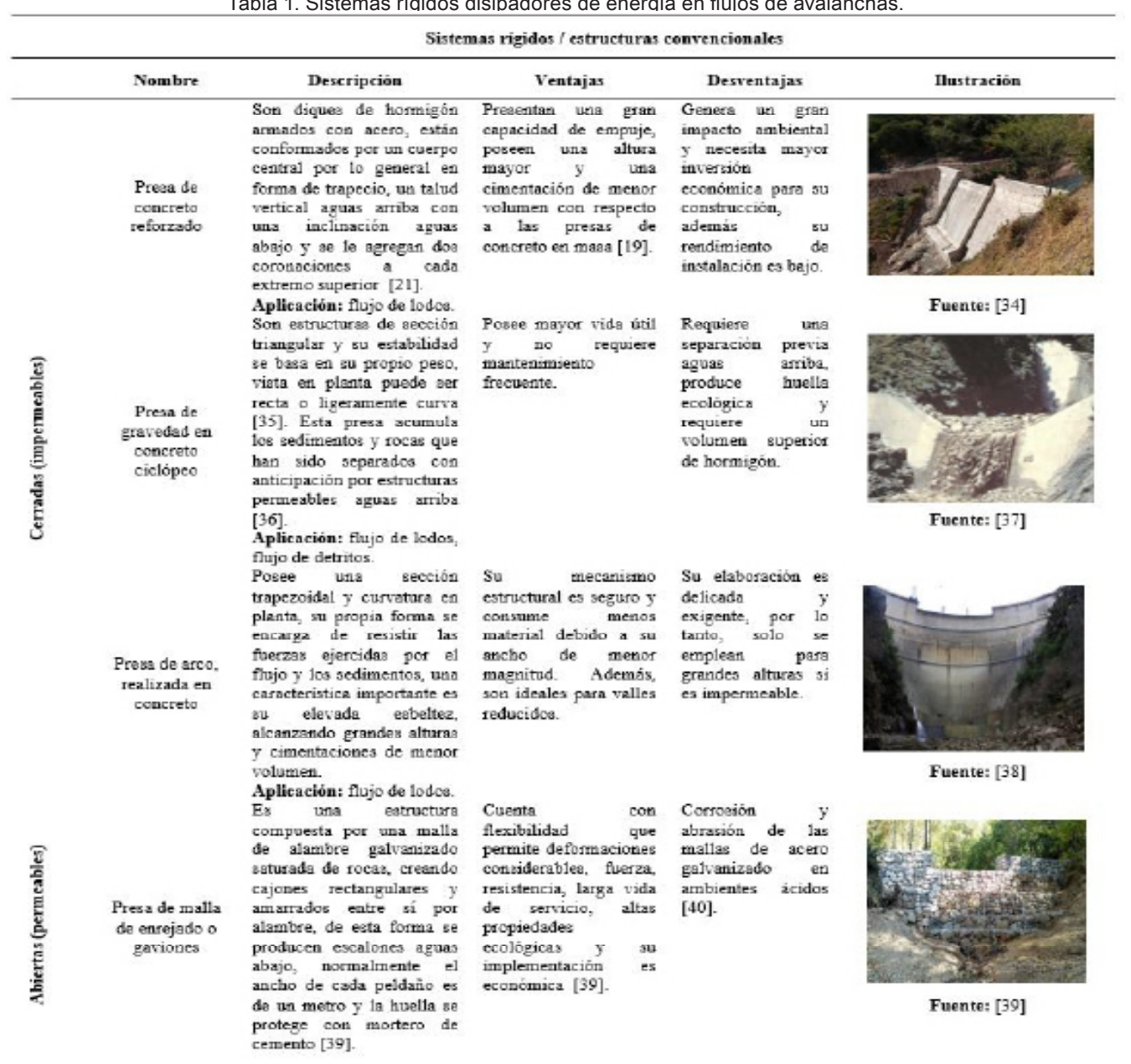




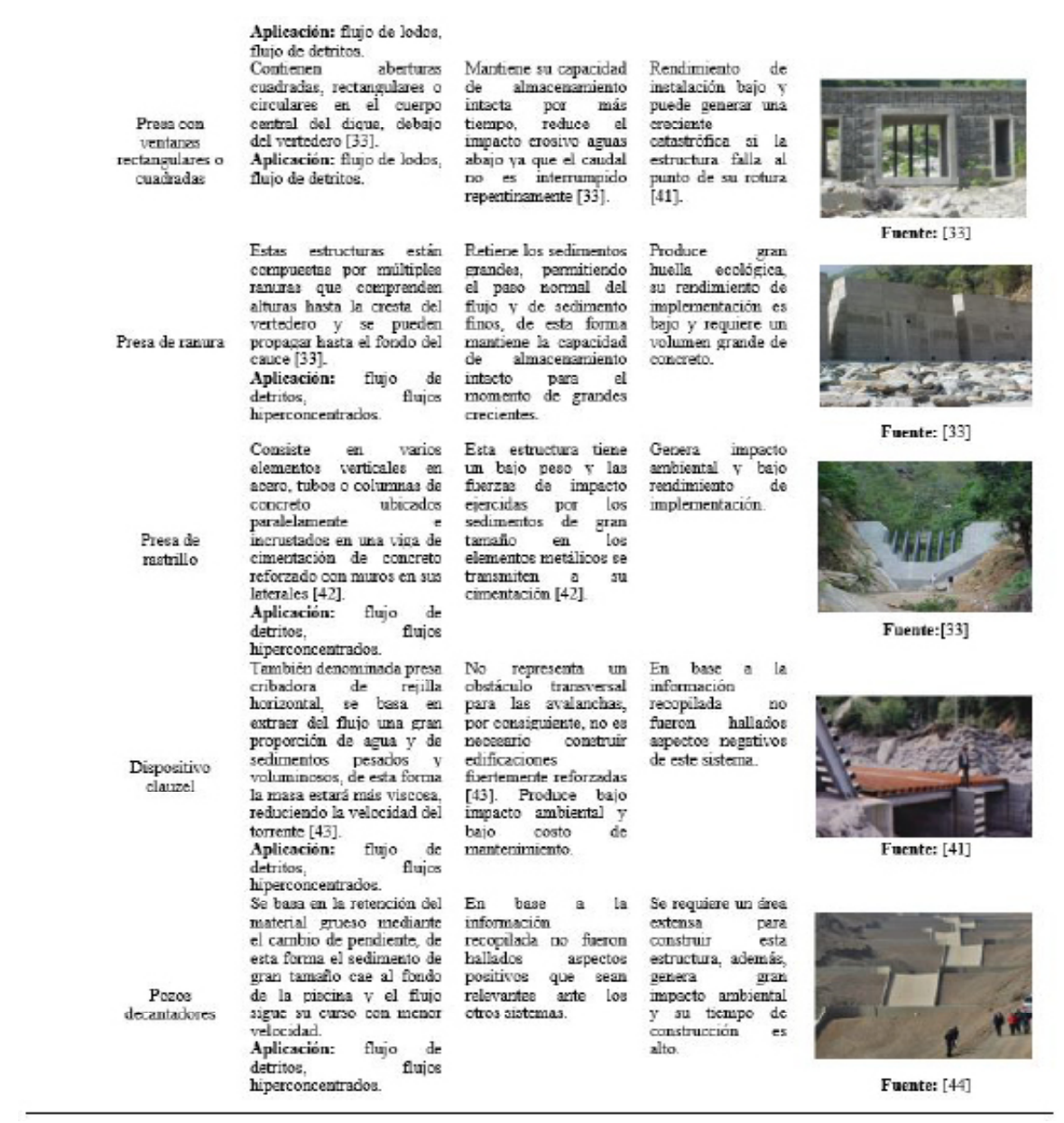

\section{Estructuras flexibles}

Las barreras flexibles son estructuras que permiten grandes deformaciones, ya que están compuestas por una red de anillos entrelazados en acero de alta resistencia, así mismo, estas características le proporcionan una gran capacidad para soportar cargas estáticas y dinámicas.

Tabla 2. Sistemas flexibles disipadores de energía en flujos de avalanchas.

\begin{tabular}{|c|c|c|c|c|}
\hline \multicolumn{5}{|c|}{ Sistemas flexibles / estructuras innovadoras } \\
\hline Nombre & Descripción & Ventajas & Desrentajas & Ilustración \\
\hline $\begin{array}{c}\text { Barrera } \\
\text { dinámica } \\
\text { contra } \\
\text { avalanchas }\end{array}$ & $\begin{array}{l}\text { Su cuerpo central está conformado por } \\
\text { una malla flexible en forma de anillos en } \\
\text { acero, anclada al fondo y a los dos } \\
\text { taludes de la sección transversal para } \\
\text { transmitir las tensiones al terreno [21]. } \\
\text { Aplicación: Flujo de lodos, flujo de } \\
\text { detritos. } \\
\text { Empresas en este mercado: Geobrueg } \\
\text { Warco S.A.S. Maccaferri, Trumer } \\
\text { Schutzbauten, Pfeifer Isofer. }\end{array}$ & $\begin{array}{l}\text { Representan un } \\
\text { ahorro considerable } \\
\text { en costo y tiempo de } \\
\text { instalación, no } \\
\text { requieren } \\
\text { cimentaciones } \\
\text { grandes solo } \\
\text { anclajes, además, se } \\
\text { mimetizan dentro } \\
\text { del entorno. }\end{array}$ & $\begin{array}{l}\text { Requicre } \\
\text { mantenimiento } \\
\text { constante para su } \\
\text { respectivo vaciado y } \\
\text { limpieza, esta acción } \\
\text { se puede realizar } \\
\text { mecánicamente sin } \\
\text { embargo, de no ser } \\
\text { posible se debe } \\
\text { desmontar la barrera } \\
\text { paso a paso. }\end{array}$ & Fuente: [45] \\
\hline
\end{tabular}




\section{4}

Algunos de los lugares donde se ha implementado:

Tabla 3. Estructuras flexibles instaladas a nivel internacional y nacional [45].

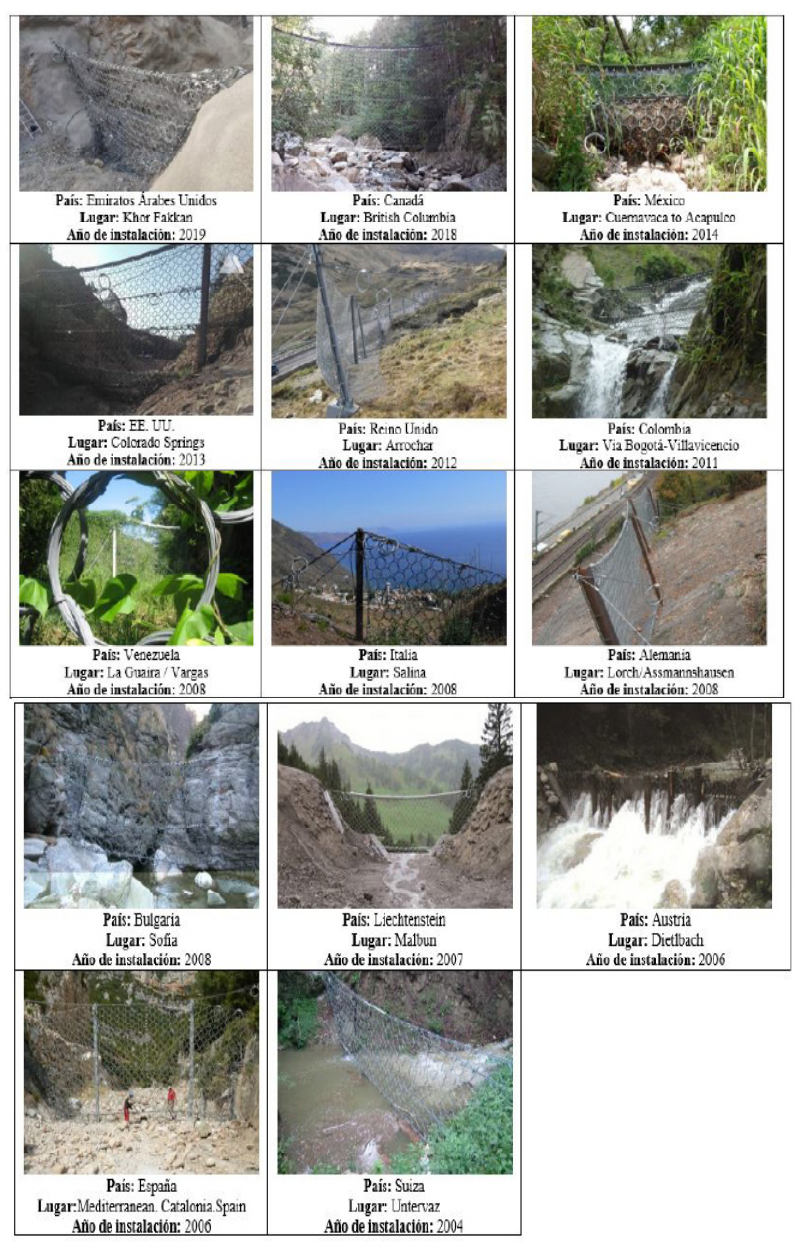

\section{Discusión}

A partir del análisis documental de los estudios realizados, se ha concretado que el tipo de flujo con mayor reincidencia en Colombia es el de detritos, con una alta tasa de mortalidad y destrucción tanto social como ambiental, causando fuertes desastres irreversibles, por lo cual se debe buscar la solución de un sistema que mejor se adapte a este tipo de fenómenos para eludir los daños graves ocasionados por este, al hacer un análisis comparativo entre las estructuras rígidas abiertas y cerradas, se tiene que lo más conveniente para los flujos de detritos son las estructuras rígidas abiertas y estructuras flexibles que no afectan el cauce cortándolo de manera perpendicular ya que los orificios permiten el paso normal del flujo y a su vez el de los sedimentos finos, mientras que, para los tipos de flujo de lodos, los sistemas más recomendables son las estructuras rígidas cerradas y abiertas con ventana. Así mismo, es conveniente utilizar estructuras flexibles, debido a que cumplen con el propósito de instalación y ofrecen un menor costo y mayor beneficio, en el siguiente grafico se puede observar dicha afirmación.

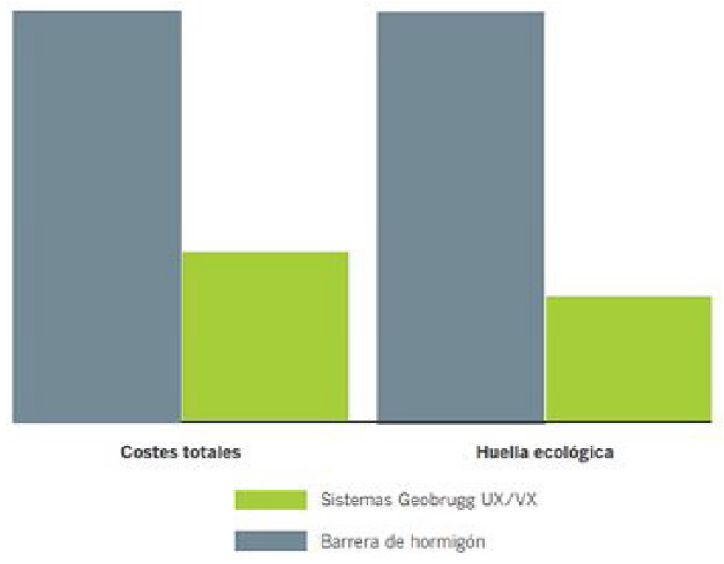

Gráfica 1. Comparación sistema rígido y flexible [45]

\section{Conclusiones}

En términos generales, con el paso del tiempo se han implementado diferentes estructuras para contrarrestar el impacto de este fenómeno catastrófico, especialmente estructuras rígidas; sin embargo, actualmente es tendencia la instalación de barreras de redes con anillos de acero de alta resistencia, debido a los beneficios que ofrece con respecto a los sistemas convencionales. A su vez, estas estructuras flexibles poseen mejores rendimientos de instalación, ya que, se componen de elementos ligeros que permiten un fácil transporte y una rápida construcción, se adaptan fácilmente a cualquier tipo de terreno representando un ahorro en costos, poseen una alta resistencia a la corrosión aumentando su durabilidad y la huella ecológica que generan en el entorno es mínima [21]. Así mismo, en el año 2016 la empresa 
Warco S.A.S. filial en Colombia de la empresa pionera a nivel mundial en prevención de riesgos geológicos (Geobrugg), implementó seis barreras dinámicas en diferentes ciudades del país. Las dos primeras están ubicadas en la quebrada Casa Teja, en la vía Bogotá- Villavicencio, poseen una capacidad de almacenamiento entre 500 y $1000 \mathrm{~m} 3$ de sedimentos, la otra red está instalada en la quebrada La Melgara - Tolima protegiendo la cabecera del municipio de las constantes crecidas de este torrente, y por último, en Suratá- Santander se realizó un proyecto que consta de tres barreras que resguardan este municipio de la quebrada Los Curos [46].

\section{Referencias}

[1] V. Gibiat y E. Plaza, "Estudio experimental de emisiones acústicas precedentes a avalanchas granulares", Interciencia: Revista de ciencia y tecnología de América, vol. 35, no. 11, pp 807-811, 2010

[2] D. Castro, J.P. Mery, R. Aravena y C. Sanhueza, "Técnicas para contener el manto de nieve en la zona de inicio de avalanchas", Revista de la Construcción, vol. 9, no. 2, pp. 39-52, dic. 2010

[3] M. Ortuño y P. Roig, P, "La defensa contra los riesgos naturales. El ejemplo de los aludes de nieve", Enseñanza de las Ciencias de la Tierra, vol. 13, no. 2, pp. 114-126, 2017

[4] A. Castelló, "Avalanchas: Estudio fisico medico", Apunts Medicina de L'Esport, vol. X, no. 38 , pp. $75-88,1973$

[5] A. Rodríguez Valdivia, A. Tapia Tosetti y C. AlbornozEspinoza, "SUSCEPTIBILIDADDE MOVIMIENTOS EN MASA EN EL VALLE DE SOCOROMA, PRECORDILLERA ANDINA DE ARICA Y PARINACOTA", Diálogo Andino, no.44, 2014
[6] D.A. Gómez-Cortés, “Medición de los esfuerzos generados por un Flujo de detritos sobre una superficie plana", tesis de maestría, Universidad Politecnica de Catalunya, Barcelona, España, 2012

[7] L. Vargas Garay, O. D. Torres Goyeneche, y G. A. Carrillo Soto, "Evaluación del modelo de hidrograma unitario SCS en la determinación de caudales máximos en cuencas de Norte de Santander", Respuestas, vol. 24, no 1, pp. 6-15, ene. 2019

[8] S. Moretti, S. Cuomo y S. Aversa, "Feasibility of Foothill Barriers to Reduce the Propagation of Debris Avalanches", En: Geotechnical Research for Land Protection and Development, Suiza: Springer, 2019.

[9] C. García-Hernández, J. Ruiz-Fernandez, C. Sánchez-Posada, S. Pereira-Paulo y M. Oliva, "An extreme event between the little ice age and the 20th century: the snow avalanche cycle of 1888 in the asturian massif (Northern Spain)", Cuadernos de Investigación Geográfica, vol. 44, no. 1 pp. 187-212, 2018

[10] J. Suárez, Deslizamientos: análisis geotécnico. Tomo. 1. Peru: Civilgeeks

[11] M. Ferrer-Gijon, "Deslizamientos, desprendimientos, flujos y avalanchas", En: Riesgos Geologicos, Madrid: I.G.M.E, 1988.

[12] J. Suárez-Díaz, Control de erosión en zonas tropicales, Bucaramanga: Universidad Industrial de Santander, 2001

[13] P.A. Bertazzi, "Catástrofes y accidentes mayores" En: Desastres naturales y tecnológicos", España: Sumario, (s.f).

[14] C. Ramirez y G. Mery, "Las avalanchas en Chile: Efectos y sistemas de control", Revista de la Construcción, vol. 6, no. 1, pp. 48-63, 


\section{6}

2007

[15] A. Sepúlveda, J. Patiño-Franco y C.E. Rodríguez-Pineda, "Metodología para evaluación de riesgo por flujo de detritos detonados por lluvias: caso Útica, Cundinamarca, Colombia", Obras y Proyectos, vol. 20, pp. 31-43, 2016

[16] Universidad EAFIT, Universidad del Valle OSSO, "Desastres de origen natural en Colombia 1979 - 2004", Medellin: Mechelin Hermelin, 2005

[17]P.N. Valencia, "Por invierno, San Carlo vive drama sin fin," El Tiempo, 1990. [En línea]. Disponible en: https://www.eltiempo.com/ archivo/documento/MAM-64383

18] J. González y A. Buendía, "Devastación del río Páez: 25 años contra la desmemoria," Co.marca Periodismo Universitario, 2019 [En línea]. Disponible en: https://comarcadigital.com/ escritos-periodisticos/879-devastacion-del-riopaez-25-anos-contra-la-desmemoria, 2019.

[19]N J. Suárez-Díaz, "Visión Geotécnica de la Amenaza Sísmica. Con énfasis en el Nororiente de Colombia y la ciudad de Bucaramanga". Erosion.com.co, eotecnología S.A.S. [En línea]. Disponible en: http://www.erosion.com. co/efectos-del-agua-en-deslizamientos/43deslizamiento-e-inundaci\%C3\%B3n-engir\% $\%$ 3\%B3n-santander.html

[20] D. Rivera-Marín, "El país sumido en la tristeza por el desastre de Salgar," Semana, 2015. [En línea]. Disponible en: https://www. semana.com/nacion/articulo/avalancha-ensalgar-deja-48-muertos-un-centenar-dedesaparecidos/428243-3

[21]A. González-Fadrique, "Estudio de inudabilidad de la Finca de Santa Leocadía en Cillorigo de Liébana", trabajo de grado, Universidad de Cantabria, Santande, España, 2019.
[22] G. Fiebiger, "The Estimation of the Hazard Potency of Debris Flows and The Step to Step Method", En: Environmental Forest Science, Kyoto. Suiza:Springer, 1998.

[23] J.A. Guirao-Goris, A. Olmedo-Salas y E. Ferrer-Ferrandis, "El artículo de revisión," Revista Ibero-americana de Enfermagem Comunitaria, 2008. [En línea]. Disponible en: https://www.uv.es/joguigo/valencia/Recerca files/el_articulo_de_revision.pdf

[24] J.M. Ortiz-García, "Guía descriptiva para la elaboración de protocolos de investigación," Salud en Tabasco, vol. 12, no 3, pp. 530-540, 2006

[25] E.V. Llerena-Oña, "Análisis técnico para la ubicación de un sistema de mallas flexibles de retención de sólidos de flujo de lodos del Volcán Cotopaxi,"tesis de pregrado, Universidad de las Fuerzas Armadas, Ecuador, 2016

[26] R. López-Alonso, "Determinación de la relación entre calado y caudal en flujos hiperconcentrados", Foresta, no. 23, pp. 54-60, 2003

[27] M.S. Páez, S.M. Moreiras, A. Brenning y L.B. Giambiagi, "Flujos de detritos y aluviones históricos en la cuenca del río Blanco (3255'$33^{\circ} 10^{\prime}$ y $\left.69^{\circ} 10^{\prime}-69^{\circ} 25^{\prime}\right)$, Mendoza" Revista de la Asociación Geológica Argentina, vol. 70, no. 4, pp. 488-498, 2013

[28] "Flujo delodos",2010. [En línea]. Disponible en: http://mirian-yumbillo.blogspot.com/2010/05/ flujos-de-lodo.html

[29] A. Peña-Jumpa, "Lluvias torrenciales, Huaycos, Inundaciones y Derechos en el Perú," SERVINDI - Servicios en comunicación Intercultural, 20 Marzo 2017. [En línea]. Disponible en: https://www. servindi.org/actualidad-opinion/17/03/2017/ lluvias-torrenciales-huaycos-inundaciones-y- 
derechos-en-el-peru

[30] Mundo, "Confirma Santos 210 muertos tras avalancha en Colombia," El Siglo de Coahuila, [En línea]. Disponible en: http://www.elsiglocoahuila.mx/coahuila/ noticia/135997.confirma-santos-210-muertostras-avalancha-en-colombia.html

[31] Pixabay, Avalanchas de nieve. [En línea]. Disponible en: https://pixabay.com/es/photos/ monta $\% \mathrm{C} 3 \% \mathrm{~B} 1 \mathrm{a}-\mathrm{avalanch}$-nieve-montblanc-4254821/

[32] D.Z. Pivalica Cisternas, "Universidad Andres Bello," 2017. [En línea]. Disponible en: http:// repositorio.unab.cl/xmlui/handle/ ria/5017

[33] J.L. López Sánchez, "Respuesta orfodinámica a la construcción de presas de retención de sedimentos en ríos de montaña" tesis de pregrado. Caracas: Academia Nacional de la Ingeniería y el Hábitat, Caracas, 2014

[34]MoreliActiva.com, "Construye Sedrua seis presas ciclópeas en Michoacán”, 2018. [En línea]. Disponible en: https://moreliactiva. com/construye-sedrua-seis-presas-ciclopeasen-michoacan/

[35] P.A. Obando-Calderón, "Análisis geotécnico de la fundación de presas de gravedad: aplicación al Proyecto Hidroélectrico Brasil”, tesis de pregrado. Universidad de Costa Rica, 1999

[36] E. Ramos-Jiménez , A. Echavarría-Luna, M. Á. Cruz-Guerrero y I. Domínguez-Jaimes, "Propuesta para Implementación de Obras de Retención de Escombros en la Parte NW del Volcán Popocatépetl'. Instituto de Protección Civil del Estado de México, (s.f).

[37] J.L. García-Rodríguez, "Obras fluviales en cauces torrenciales," En: III SEMINARIO
INTERNACIONAL DE POTAMOLOGÍA "JOSÉ ANTONIO MAZA ÁLVAREZ", Tuxtla Gutiérrez, 2011. [En línea]. Disponible en: https://www.yumpu.com/es/document/ view/31181885/obras-fluviales-en-caucestorrenciales

38] La Asociación de Ingenieros de Caminos, Canales y Puertos y de la Ingeniería Civil, "La visión para la ingeniería civil en 2025". [En línea]. Disponible en: http://ropdigital.ciccp.es/ pdf/publico/2010/2010_septiembre_3513_05. pdf

[39] E.A. Chiel Díaz, "Guía técnica para el diseño y construcción de una presa de gaviones," trabajo de grado, Universidad Autónoma Agraria Antonio Narro Coahuila, México, 2015

[40] J. Suárez Díaz, "Los Gaviones,” de Control de erosión en zonas tropicales, Bucaramanga, U. Industrial de Santander, 2001, pp. . [En línea]. Disponible en: https:// www.erosion.com.co/ control-de-erosion-en- zonas-tropicales.html

[41] N.J. Cely Calixto, Diseño para obras para la retención de lavas torrenciales sobre la quebrada la calderera, municipio de Gramalote, Norte de Santander, 2014

[42]M. Mengual Fernández, "Scribd," [En línea]. Disponible en: https://es.scribd.com/ document/352273786/Presas-Rastrillo.

[43]R. López-Alonso, "Hidrotecnias para la gestión de acarreos en cauces de montaña," Foresta, vol. 3, no. 8, pp. 16-19, 1999

[44] DIPRES. Dirección de presupuestos de Goberno de Chile "Gobierno Regional Región de Antofagasta," 2013. [En línea]. Disponible en: http://desarrollo2.applicatta.cl/ goreantofagasta/stat/api_anteriores/index.php/ noticias/leer noticia? $\mathrm{id}=\mathbf{7 2 5}$ 


\section{8}

[45] Geobrugg, "Geobrugg brugg,” [En línea]. Disponible en: https://www.geobrugg.com/es/ Sobre-nosotros-44608,7864.html

[46]J.M. Hernández-Bonilla, “¿Es posible prevenir catástrofes similares a la de Mocoa?," El Espectador, [En línea]. Disponible en: https:// www.elespectador.com/economia/es-posibleprevenir-catastrofes-similares-la-de-mocoaarticulo-689277, 2017 